Pacific Journal of Mathematics

UNIVERSAL OBSERVABILITY AND CODIMENSION ON
SUBGROUPS OF BORED SUBGROUPS

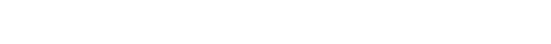




\title{
UNIVERSAL OBSERVABILITY AND CODIMENSION ONE SUBGROUPS OF BOREL SUBGROUPS
}

\author{
JOHN BRENDAN SULLIVAN
}

\begin{abstract}
A subgroup $H$ of an affine algebraic group $G$ is observable in $G$ if the quotient variety $G / H$ is quasi-affine (equivalently, if each character on $H$ is the character of a one-dimensional $H$-submodule of an irreducible $G$-module). The question is how to characterize the universally observable groups, i.e., those which are observable in every group in which they can be embedded. We remark that the relation that $G / H$ should be affine for every embedding of $H$ is equivalent to $H$ being reductive, by work of Cline, Parshall, Scott. A sufficient condition for the universal observability of a solvable group is that a certain monoid of characters for the inner operation of $H$ on its hyperalgebra should be a group. Here, we give a two-dimensional example (a codimension one subgroup of a Borel subgroup of $\mathrm{GL}_{2}$ ) to show that this sufficient condition is not necessary. Secondly, we give a method for testing a group for the failure of universal observability, which we use to show the non-universal observability of a famly of codimension one subgroups of Borel subgroups of $\mathrm{GL}_{n}(n \geq 3)$. We remark that the universal observability of an affine algebraic group is equivalent to the universal observability of its solvable radical. Consequently, we only need to sort the solvable groups for those that are universally observable.
\end{abstract}

Introduction. An affine algebraic group $H$ is called universally observable if every embedding of $H$ in an affine algebraic group $G$ gives a quasi-affine quotient $G / H$ (cf. [1]). Here we study aspects of universal observability for solvable groups which are codimension one subgroups of Borel subgroups of $\mathrm{Gl}_{n}$ or $\mathrm{Sl}_{n}$.

We use two methods to establish the universal observability of certain groups. (1) Sweedler's method: The inner operation of $H$ on its hyperalgebra generates a monoid of characters (see $\$ 1$ ). That this monoid should be a group is a sufficient condition for universal observability of a solvable group. (2) Direct calculation for two-dimensonal groups for which the first method fails (see §2). This work began as a study of whether the sufficient condition (1) for universal observability was also necessary. Our principal results are as follows. (1) Using the first method, we show that Ker $\chi$ is universally observable when $\chi: B \rightarrow G_{m}$ is a character in the dominant chamber of a Borel subgroup $B$ of $\mathrm{Sl}_{n}$. (2) In characteristic zero, we show the universal observability of the semidirect product $G_{a} \times{ }_{w} G_{m}$, where the 
multiplicative group operates on the additive group by the $w$ th power character $(w>2)$. These groups provide a negative answer to the question of whether Sweedler's sufficient condition is also necessary.

There is something singular about the appearance of the universally observable groups $G_{a} \times{ }_{w} G_{m}$. In fact, $G_{a} \times{ }_{w} G_{m}$ is a codimension one subgroup of a Borel subgroup of $\mathrm{Gl}_{2}$, but when we look for codimension one subgroup of a Borel subgroup of $\mathrm{Gl}_{2}$, but when we look for codimension one subgroups of Borel subgroups of $\mathrm{Gl}_{n}(n>2)$ which are analogously situated and universally observable, we find none (see §3). Our method for looking for the failure of universal observability for solvable subgroups $H$ of $\mathrm{Gl}_{n}$ (considered as an $n$-dimensional representation of $H$ ) is to reposition $H$ in $\mathrm{Gl}_{n}$ by tensoring the representation with a character on $H$. We detect a change in the geometry of the quotient by using the following theorem from [4]. For a character $\chi$ on a Borel subgroup $B$ of $\mathrm{Sl}_{n}$, the quotient $\mathrm{Sl}_{n} / \operatorname{Ker} \chi$ is quasi-affine exactly when $\pm \chi$ lies in the dominant chamber of $B$. Repositioning attempts to move a character from one chamber into a different chamber.

This work also suggests the possible modification of the definition of universal observability to be that a group $H$ is u.o.* if, for every morphism $H \rightarrow G$ with finite kernel, the quotient of $G$ by the image of $H$ is quasi-affine. $G_{a} \times{ }_{w} G_{m}$ is not u.o. ${ }^{*}$, since it is isogeneous to $G_{a} \times{ }_{1} G_{m}$, a Borel subgroup of $\mathrm{PGl}_{2}$, which is not universally observable. This would open up the question of Sweedler to further work.

I offer the following question here. Let $H$ be a subgroup of $\mathrm{Gl}(V)$. Suppose that, for every character $\chi$ on $H, H \rightarrow \mathrm{Gl}(V \otimes \chi)$ gives a quasi-affine quotient of $\mathrm{Gl}(V \otimes \chi)$ by the image of $H$. Is $H$ then universally observable?

I benefited from discussions with John Ballard in the course of the work on $\$ 2$.

The last remark in the abstract is included at the thoughtful suggestion of the referee. Please see Theorems 9 and 10 of [1] to establish the fact stated.

\section{The hyperalgebra and universal observability. ${ }^{1}$}

1.1. Let $H$ be an affine algebraic group defined over a field $K$. Let $X(H)$ be the character group of $H$. The inner operation of $H$ on itself induces an operation of $H$ on the coordinate ring $A_{H}$ and dually, on the hyperalgbra hy $(H)$. See [3] for the definition of the hyperalgebra.

\footnotetext{
${ }^{1}$ The material in this section is adapted from unpublished notes of M. Sweedler [5].
} 
Definition. $C(H)$ is the smallest family of finite-dimensional $H$ modules which contains all finite-dimensional submodules of hy $(H)$ and which is closed under the operations of taking submodules, direct sums, and exterior, symmetric and tensor powers.

Definition. $S$ is the monoid of those characters on $H$ which are characters of one-dimensional modules in $C(H)$.

\subsection{LeMma. $C(H)$ is closed under quotients when $S$ is a group.}

Proof. Let $U$ be a submodule (of some dimension $m$ ) of a module $V$ in $C(H) . U, \wedge^{m} U, \Lambda^{m} V$ are modules in $C(H)$, and the character $\chi$ of the one-dimensional module $\wedge^{m} U$ is an element of $S$. Since the kernel of the canonical map $V \otimes \wedge^{m} U \rightarrow \wedge^{m+1} V$ is $U \otimes \wedge^{m} U,(V / U) \otimes \wedge^{m} U$ is isomorphic to the image of $V \otimes \wedge^{m} U$ in $\wedge^{m+1} V$, which is an element of $C(H)$.

Let $W$ be a one-dimensional module in $C(H)$ with character $\chi^{-1}$. Then $V / U$ is isomorphic to $(V / U) \otimes \wedge^{m} U \otimes W$, which in turn is isomorphic to an element of $C(H)$. This completes the proof.

1.3. LeMMA. Let $H$ be a subgroup of a group $G$. Each module in $C(H)$ is an $\mathrm{H}$-submodule of a module in $C(G)$.

Proof. The inclusion $H \hookrightarrow G$ induces the inclusive morphism of $H$-modules $i: \operatorname{hy}(H) \rightarrow \operatorname{hy}(G)$. If $M$ is a finite-dimensional submodule of hy $(H)$, then $i(M)$ is an $H$-submodule of a finite-dimensional $G$-submodule of hy $(G)$ since hy $(G)$ is locally finite. This property of hy $(H)$ relative to hy $(G)$ evidently extends to the property of $C(H)$ relative to $C(G)$ stated in the Lemma.

1.4. Proposition. Let $H$ be solvable and connected. $H$ is universally observable if $S$ is a group.

Proof. $H$ is the semidirect product of its unipotent radical $U$ and any maximal torus $T$. The character group $X(T)$ is a direct product $S_{1} \times S_{2}$, where $S_{1}$ contains the restriction of $S$ to $T$ as a subgroup of finite index. Correspondingly, $T$ is the direct product $T_{1} \times T_{2}$ where $S_{1}$ is trivial on $T_{2}$ and $S_{2}$ is trivial on $T_{1}$.

First, we show that $T_{2}$ centralizes $H$. The following conditions are equivalent: (1) $T_{2}$ centralizes $H$. (2) $T_{2}$ operates trivially on $A_{H^{*}}$ (3) $T_{2}$ 
operates trivially on hy(H). (4) $T_{2}$ operates trivially on all 1-dimensional $H$-module quotients of finite-dimensional $H$-submodules of hy $(H)$. The equivalences are all immediate. The equivalence of (3) and (4) uses the facts that $H$ is solvable and that $T$ operates linear reductively.

By Lemma 1.2, $C(H)$ contains the one-dimensional quotients of finite-dimensional submodules of hy $(H)$. Hence, the characters of these modules lie in $S$ and are trivial on $T_{2}$. By the equivalence of (1) and (4), $T_{2}$ centralizes $H$, and so, $H=\left(U T_{1}\right) \times T_{2}$ and $X(H)=X\left(U T_{1}\right) \times X\left(T_{2}\right)$.

Let $H \rightarrow G$ be any embedding. For any character $\chi_{2} \in X\left(T_{2}\right), K \chi_{2}$ is a $T_{2}$-submodule of some finite-dimensional $G$-module $V$. Let $W$ be the $H$-submodule of $V$ generated by $\mathrm{K \chi}_{2}$. The central subgroup $\mathrm{T}_{2}$ of $\mathrm{H}$ operates on $W$ by the character $\chi_{2}$.

Let $n$ be the index of $S$ in $S_{1}$ above, and let $m$ be the dimension of $W$. The character for the operation of $H$ on $\wedge^{m} W\left(\hookrightarrow \wedge^{m} V\right)$ has the form $\left(\chi_{1}, m \chi_{2}\right)$, where $\chi_{1}$ is a charcter on $U T_{1} .-n \chi_{1}$ lies in $S$, and $K\left(-n \chi_{1}\right)$ is an $H$-submodule of some $G$-module, by Lemma 1.3. Since $K\left(n\left(\chi_{1}, m \chi_{2}\right)\right)$ is an $H$-submodule of some $G$-module $\left[K\left(\chi_{1}, m \chi_{2}\right)\right.$ already has this property], so is $K\left(-n \chi_{1}\right) \otimes K\left(n\left(\chi_{1}, m \chi_{2}\right)\right)=K\left(1, n m \chi_{2}\right)$. Therefore, for any $Y_{1} \in X\left(U T_{1}\right)$ and $\chi_{2} \in X\left(T_{2}\right), K\left(m n\left(Y_{i}, \chi_{2}\right)\right)$ is a $H$-submodule of some $G$-module (where $m$ depends on $\chi_{2}$ ). By the observation [1, Th. 9], $H$ is observable in $G$.

2. We present a class of solvable connected universally observable groups $H$ whose monoid $S$ of characters arising in $C(H)$ is not a group.

Let $K$ be an algebraically closed field of characteristic zero and $w$ an integer greater than 2. Let $H$ be the semidirect product $G_{a} \times{ }_{w} G_{m}$, where the multiplicative group $G_{m}$ operates on the additive group $G_{a}$ via the $w$ th-power character.

THEOREM. $H=G_{a} \times{ }_{w} G_{m}$ is universally observable, but the monoid of characters of $H$ in $C(H)$ is not a group.

Proof. Universal observability is shown in \$2.3. Here we prove the part dealing with the monoid $S$ of characters. Evidently, $S$ is contained in the monoid generated by the characters appearing in the operation of $G_{m}$ on hy $(H)$. Since $G_{m}$ operates trivially on hy $\left(G_{m}\right)$, the latter monoid coincides with the monoid of characters of $G_{m}$ on hy $\left(G_{a}\right)$, which equals $\left\{n w \mid n \in \mathbf{Z}_{\geq 0}\right\}$. This monoid contains only the trivial group as a subgroup.

REMARK. In order to show that $H=G_{a} \times{ }_{w} G_{m}(w>2)$ is universally observable, we need only consider embeddings $H \rightarrow G$ where $G$ is a 
general linear group $\mathrm{Gl}_{n}, n \in \mathbf{Z}_{>0}$. In fact, if $H \rightarrow G$ is any embedding, then we may embed $G$ in some $\mathrm{Gl}_{n}$. If $\mathrm{Gl}_{n} / H$ is quasi-affine, so is $G / H$.

2.1. Let $\mathrm{Gl}_{n}$ be the general linear group over an algebraically closed field $K$ of characteristic zero. $B, U$, and $T$ are the upper triangular, upper unipotent, and diagonal subgroups, and $\mathrm{gl}_{n}$ is the Lie algebra of $n \times n$ matrices over $K$. We work with the adjoint representation of $\mathrm{Gl}_{n}$ on $\mathrm{gl}_{n}$.

Let $A=\left(A_{i j}\right)$ be a non-zero nilpotent $n \times n$ matrix in Jordan form

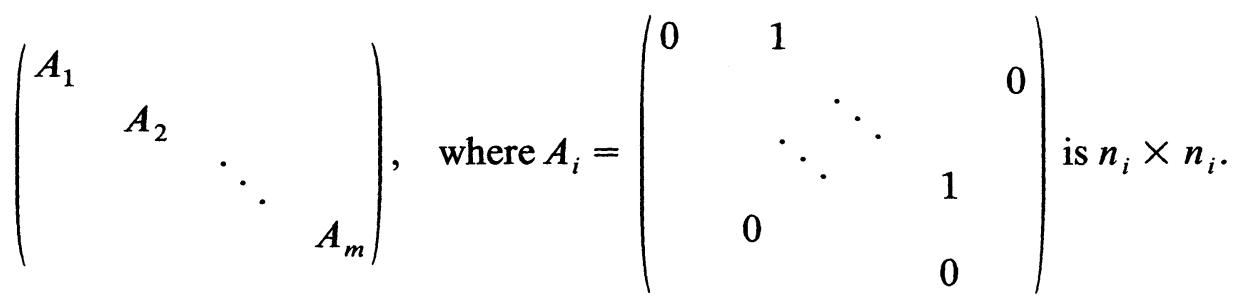

Let $C_{A}$ be the subgroup of $\mathrm{Gl}_{n}$ of elements that act trivially on $A$ (i.e., the centralizer of $A$ ), and let $D_{A}$ be the subgroup of diagonal elements of $C_{A}$.

LEMMA. $D_{A}$ is a maximal torus of $C_{A}$.

Proof. Let

$$
I=\left\{1<i<n \mid A_{i, i+1}=0\right\}=\left\{i_{1}<i_{2}<\cdots<i_{m-1}\right\} .
$$

$C_{A}$ is the group

$$
\begin{aligned}
& \left\{\left(a_{i j}\right) \in \mathrm{Gl}_{n} \mid a_{i, k-1} A_{k-1, k}=A_{i, i+1} a_{i+1, k} ; 1 \leq i, k \leq n\right\} \\
& =\left\{\left(a_{i j}\right) \in \mathrm{Gl}_{n} \mid a_{i k} A_{k, k+1}=A_{i, i+1} a_{i+1, k+1} ;\right. \\
& 1 \leq i \leq n, 0 \leq k \leq n-1\} .
\end{aligned}
$$

Here and below, a term $a_{i k}$ or $A_{i k}$ is understood to be zero if $i$ or $k$ equals 0 or $n+1$.

$C_{A}$ is the set of all $\left(a_{i j}\right)$ satisfying $\mathrm{c} 1, \mathrm{c} 2, \mathrm{c} 3$ for $1 \leq i \leq n$, and $0 \leq k \leq n-1$.

c1. $a_{i k}=a_{i+1, k+1}$ when $i, k \notin I$.

c2. $a_{i+1, k+1}=0$ when $i \notin I$ and $k \in I$.

c3. $a_{i k}=0$ when $i \in I$ and $k \notin I$.

$D_{A}$ is the subgroup $\left\{\left(a_{i j}\right) \in T \mid a_{i i}=a_{i+1, i+1}\right.$ for $\left.i \notin I\right\}$, a torus of dimension $m$. In order to prove the lemma, it will suffice to show that $D_{A}$ is a maximal torus in $\operatorname{Cent}\left(D_{A}\right) \cap C_{A}$, where $\operatorname{Cent}\left(D_{A}\right)$ is the centralizer of $D_{A}$ in $\mathrm{Gl}_{n}$. We do this by showing that $\operatorname{Cent}\left(D_{A}\right) \cap C_{A}$ is upper triangular, and by noting that, since $D_{A}$ already contains the diagonal piece of any upper triangular element of $C_{A}, B \cap C_{A}$ equals $D_{A} \cdot\left(U \cap C_{A}\right)$. 
Since $K$ is an infinite field,

$$
\operatorname{Cent}\left(D_{A}\right)=\left\{\left(\begin{array}{ccc}
B_{1} & & \\
& \ddots & \\
& & B_{m}
\end{array}\right) \mid B_{i} \text { is an invertible } n_{i} \times n_{i} \text { matrix }\right\} .
$$

Take any element

$$
\left(a_{i j}\right)=\left(\begin{array}{ccc}
B_{1} & & 0 \\
& \ddots & \\
0 & & B_{m}
\end{array}\right) \text { from } \operatorname{Cent}\left(D_{A}\right) \cap C_{A} .
$$

(1) $B_{1}$ is upper triangular. In fact, for $i<i_{1}$ and $k=0$, application of cl to $\left(a_{i j}\right)$ gives the relation $0=a_{i 0}=a_{i+1,1}$; and by cl, for $i, k<i_{1}$, $a_{i k}=a_{i+1, k+1}$. Hence, $a_{i k}=0$ for $0<k<i \leq i_{1}$.

(2) $B_{k}$ is upper triangular for $1<k \leq m$. The first row (column) of $B_{k}$ is the $i_{k}+1$ row (column) of $\left(a_{i j}\right)$. By c2, for $i_{k}<j<i_{k+1}, a_{j+1, i_{k}+1}=0$; by repeated use of cl, $a_{j+j^{\prime}, i_{k}+j^{\prime}}=0$ for $i<j^{\prime} \leq i_{k+1}-j$. Hence, $B_{k}$ is upper triangular.

2.2. Let $N_{A}$ be the normalizer of $K A$ in $\mathrm{Gl}_{n}$ (the stabilizer of $K A$ under the adjoint representation). $N_{A}$ operates on $K A$ by some character $\chi$ whose kernel is $C_{A}$. Let $H^{\circ}$ denote the connected component of 1 in a group $H$.

LEMMA $\left(N_{A} \cap T\right)^{\circ}$ is a maximal torus of $N_{A}$.

Proof. Since the codimension of $C_{A}$ in $N_{A}$ is one at most, the codimension of $D_{A}$ in a maximal torus of $N_{A}$ is one at most. We show the maximality of $\left(N_{A} \cap T\right)^{\circ}$ by showing that $D_{A}$ indeed has codimension one in $\left(N_{A} \cap T\right)^{\circ}$. Define $\varphi: G_{m} \rightarrow\left(N_{A} \cap T\right)^{\circ}$ by

$$
\varphi(t)=\left(\begin{array}{ccc}
B_{1} & & \\
& \ddots & \\
& & B_{m}
\end{array}\right),
$$

where $B_{i}$ is the diagonal matrix

$$
\left(\begin{array}{lll}
t & & \\
& t^{2} & \\
& \ddots & \\
& & \cdot t^{n_{i}}
\end{array}\right)
$$

Since $\varphi(t) A \varphi(t)^{-1}=t^{-1} A, \varphi\left(G_{m}\right) \cap D_{A}$ is finite and one is the codimension of $D_{A}$ in $D_{A} \varphi\left(G_{m}\right)$. 
2.3. TheOREM. Let $K$ be algebraically closed of characteristic zero. $G_{a} \times{ }_{w} G_{m}$ is universally observable for each $w>2$.

Proof. By the remark at the end of $\S 2$, we need only show that each embedding $\varphi: G_{a} \times{ }_{w} G_{m} \rightarrow \mathrm{Gl}(V)$ determines a quasi-affine quotient $\mathrm{Gl}(V) / G_{a} \times{ }_{w} G_{m}$. The image of $L\left(G_{a}\right)$ under the differential $d \varphi$ consists of nilpotent operators on $V$, since $\varphi\left(G_{a}\right)$ is unipotent. Choose a basis for $V$ such that $d \varphi\left(L\left(G_{a}\right)\right)$ has one of its non-zero elements $A$ in Jordan form

$$
\left(\begin{array}{lllll}
A_{1} & & & \\
& A_{2} & & 0 \\
& & \cdot & \\
0 & & & A_{m}
\end{array}\right) .
$$

$G_{m}$, which operates on $G_{a}$ and $L\left(G_{a}\right)$ by the $w$-power character, operates on $d \varphi\left(L\left(G_{a}\right)\right)$ by the $w$-power character, that is, $\varphi$ maps $G_{m}$ into $N_{A}^{\circ}$, where $G_{m}$ operates on $K A$ by $t \cdot A=t^{w} A$. Let $c_{g}$ be conjugation by an element $g$ of $N_{A}^{\circ}$, mapping the torus $\varphi\left(G_{m}\right)$ into a subtorus of the maximal torus $\left(N_{A} \cap T\right)^{\circ}$ of $N_{A}$. With $c_{g} \circ \varphi$ in place of $\varphi, \varphi\left(G_{m}\right)$ is diagonal and $L\left(\varphi\left(G_{a}\right)\right)=d \varphi\left(L\left(G_{a}\right)\right)$ still equals $K A$. Therefore, we may assume that $d \varphi\left(L\left(G_{a} \times{ }_{w} G_{m}\right)\right)$ is contained by the subspace $L(T) \oplus K A$ of

$$
L\left(\mathrm{Gl}_{n_{1}} \times \cdots \times \mathrm{Gl}_{n_{m}}\right)=\mathrm{gl}_{n_{1}} \times \cdots \times \mathrm{gl}_{n_{m}} \quad\left(\subset \mathrm{gl}_{n}=L(\mathrm{Gl}(V))\right) .
$$

By Theorem 8.6 of [2], $\varphi\left(G_{a} \times{ }_{w} G_{m}\right)$ is contained by $\mathrm{Gl}_{n_{1}} \times \cdots \times \mathrm{Gl}_{n_{m}}$ $\subset \mathrm{Gl}_{n}$.

Recall that $X(G)$ is the group of characters of a group G. (1) If $\mathrm{Sl}_{n_{1}} \times \cdots \times \mathrm{Sl}_{n_{m}}$ does not contain $\varphi\left(G_{a} \times{ }_{w} G_{m}\right)$, then the morphism

$$
\begin{aligned}
G_{a} \times{ }_{w} G_{m} & \stackrel{\varphi}{\rightarrow} \mathrm{Gl}_{n_{1}} \times \cdots \times \mathrm{Gl}_{n_{m}} \\
& \stackrel{\pi}{\rightarrow} \frac{\mathrm{Gl}_{n_{1}} \times \cdots \times \mathrm{Gl}_{n_{m}}}{\mathrm{Sl}_{n_{1}} \times \cdots \times \mathrm{Sl}_{n_{m}}} \cong G_{m} \times \cdots \times G_{m}
\end{aligned}
$$

determines (dually) a non-trivial subgroup $(\pi \circ \varphi) *\left(X\left(G_{m} \times \cdots \times G_{m}\right)\right)$ of the infinite cyclic group $X\left(G_{a} \times{ }_{w} G_{m}\right)$. Of course, the characters in this subgroup extend to characters on $\mathrm{Gl}_{n_{1}} \times \cdots \times \mathrm{Gl}_{n_{m}}$. Hence, by [1, Th. 9], $\mathrm{Gl}_{n_{1}} \times \cdots \times \mathrm{Gl}_{n_{m}} / \varphi\left(G_{a} \times{ }_{w} G_{m}\right)$ is quasi-affine. Since $\mathrm{Gl}_{n_{1}} \times \cdots \times$ $\mathrm{Gl}_{n_{m}}$ is linearly reductive, $\mathrm{Gl}_{n} / \mathrm{Gl}_{n_{1}} \times \cdots \times \mathrm{Gl}_{n_{m}}$ is affine, and so, by [1], $\mathrm{Gl}_{n} / \varphi\left(G_{a} \times{ }_{w} G_{m}\right)$ is quasi-affine. 
(2) We show that $\mathrm{Sl}_{n_{1}} \times \cdots \times \mathrm{Sl}_{n_{m}}$ does not contain $\varphi\left(G_{a} \times{ }_{w} G_{m}\right)$, and so, (1) shows that $\mathrm{Gl}_{n} / \varphi\left(G_{a} \times{ }_{w} G_{m}\right)$ is quasi-affine. If $\varphi: G_{a} \times_{w} G_{m}$ $\rightarrow \mathrm{Sl}_{n_{1}} \times \cdots \times \mathrm{Sl}_{n_{m}}$ is any (supposed) injective morphism, let $\varphi_{i}$ : $G_{a} \times{ }_{w} G_{m} \rightarrow \mathrm{Sl}_{n_{i}}$ be the $i$ th component of $\varphi$. We show that the kernel of $\varphi_{i}$ contains the $w$ th roots of unity in $G_{m}$ when $w$ is odd and the $(w / 2)$ th roots of unity when $w$ is even. Hence, $\varphi$ is not an embedding.

Let

$$
\varphi_{i}(t)=\left(\begin{array}{ccc}
t^{m_{1}} & & 0 \\
& t^{m_{2}} & \\
0 & & t^{m_{n_{i}}}
\end{array}\right) \quad \text { for } t \in G_{m} .
$$

Since $\varphi_{i}\left(G_{m}\right)$ operates on $\left(d \varphi_{i}\right)\left(L\left(G_{a}\right)\right)=K A_{i}$ by $\varphi_{i}(t) A_{i} \varphi_{i}(t)^{-1}=t^{w} A_{i}$, we have $t^{m_{j}-m_{j+1}}=t^{w}$ for $1 \leq j<n_{i}$, or in terms of exponents $m_{j}=$ $m_{1}-j w$ for $1<j \leq n_{i}$. Since $\varphi_{i}\left(G_{a} \times{ }_{w} G_{m}\right)$ consists of special linear transformations,

$$
0=\sum_{j=1}^{n_{i}} m_{i}=\sum_{j=0}^{n_{i}-1}\left(m_{1}-j w\right)=n_{i} m_{1}-\frac{\left(n_{i}\right)\left(n_{i}-1\right)}{2} w,
$$

and so $2 m_{1}=\left(n_{i}-1\right) w$.

If $w$ is odd, $w$ divides $m_{i}$, for each $i$, and $\operatorname{Ker} \varphi_{i}$ contains the $w$ th roots of unity. If $w$ is even, $w / 2$ divides $m_{i}$ for each $i$, and $\operatorname{Ker} \varphi_{i}$ contains the $(w / 2)$ th roots of unity.

2.4. Corollary. Universal observability is not a property of isogeny classes.

Proof. For any $w \neq 0$, there is the isogeny $G_{a} \times{ }_{w} G_{m} \rightarrow G_{a} \times{ }_{1} G_{m}$ mapping $(a, t)$ to $\left(a, t^{w}\right)$. For $w>2, G_{a} \times{ }_{w} G_{m}$ is universally observable, but $G_{a} \times{ }_{1} G_{m}$ is not, since it may be embedded in $\mathrm{PGl}_{2}$ as a Borel subgroup, in which case the quotient is projective.

3. We situate $G_{a} \times{ }_{w} G_{m}$ in $\mathrm{GL}_{2}$ as the kernel of a character on a Borel subgroup, and then show that there are not universally observable subgroups of $\mathrm{Gl}_{n}(n>2)$ whose situation is analogous to that of $G_{a} \times{ }_{w} G_{m}$ in $\mathrm{Gl}_{2}$.

Let $B$ be the upper triangular subgroup of $\mathrm{GL}_{2}$, and let $w$ be a positive integer. Let $\chi_{w}$ be the character on $B$ defined by

$$
\chi_{w}\left(\begin{array}{cc}
t_{1} & 0 \\
0 & t_{2}
\end{array}\right)=t_{1}^{w-1} t_{2}
$$


The kernel of $\chi_{w}$ coincides with the image of the embedding $G_{a} \times{ }_{w} G_{m} \rightarrow$ $\mathrm{Gl}_{2}$ taking $(a, t)$ to $\left(\begin{array}{cc}t & a \\ 0 & t^{1-w}\end{array}\right)$. In the second section we showed that $\operatorname{Ker} \chi_{w}$ $(w>2)$ is a universally observable group. There are two particular facts to mention about this situation. (1) Ker $\chi_{w}$ does not contain the center of $\mathrm{Gl}_{2}$; and (2) $G_{a} \times{ }_{w} G_{m}$ is isogenous to a Borel subgroup of $\mathrm{PGl}_{2}$. In contrast, for $\chi$ a character on the upper triangular subgroup of $\mathrm{Gl}_{n}$ $(n>2)$, for $\operatorname{Ker} \chi$ to be universally observable, it must, among other things, contain the center of $\mathrm{Gl}_{n}$ (Th. 3.3); consequently, it is not isogeneous to a Borel subgroup of $\mathrm{PG}_{n}$. (For $n=2$, a prescription for the universal observability of $\operatorname{Ker} \chi$ which contains the requirement that Ker $\chi$ should contain the center of $\mathrm{Gl}_{2}$ would refer only to the direct product $G_{a} \times G_{m}$.) Furthermore, we will see in the proof of the Theorem in $\$ 4$ that, for those subgroups $\operatorname{Ker} \chi$ of $\mathrm{Gl}_{n}(n>2)$ which are universally observable, the characters of Ker $\chi$ on the one-dimensional modules in $C$ $(\operatorname{Ker} \chi)$ form a group (cf. §1).

3.1. Let $K$ be an algebraically closed field of any characteristic. Fix a character $\lambda$ on $B$, the upper triangular subgroup of $\mathrm{Gl}_{n}(n>2)$, and let $i$ : $\operatorname{Ker} \lambda \rightarrow \mathrm{GL}_{n}$ be the inclusion map. We will test $\operatorname{Ker} \lambda$ for observability against those embeddings of $\operatorname{Ker} \lambda$ in $\mathrm{Gl}_{n}$ obtained from skewing $i$ by a character $\chi$ on $B$.

Let $\lambda_{i}$ be the $i$ th fundamental weight on $B$,

$$
\lambda_{i}\left(\begin{array}{ccc}
t_{1} & & 0 \\
& \ddots & \\
0 & & t_{n}
\end{array}\right)=t_{1} \cdots t_{i} .
$$

For any character $\chi=\sum_{i=1}^{n} a_{i} \lambda_{i}$, let $a_{\chi}$ stand for the "signature" $\sum_{i=1}^{n} i a_{i}$ of $\chi$. For each character $\chi: B \rightarrow \mathrm{Gl}(K \chi)$, form the representation $P_{\chi}=$ $i \otimes \chi: B \rightarrow \mathrm{Gl}\left(K^{n} \otimes K \chi\right)=\mathrm{Gl}\left(K^{n}\right)$.

LEMMA. $P_{\chi}$ is an embedding if and only if $a_{\chi}=0$.

Proof.

$$
P_{\chi}(b) \text { equals } b \cdot\left(\begin{array}{ccc}
\chi(b) & & \\
& \ddots & \\
0 & & \chi(b)
\end{array}\right), \quad \text { for } b \in B \text {. }
$$

Evidently, $P_{\chi}$ is an embedding exactly when $P_{\chi}: B \rightarrow B$ is an automorphism. Since $P_{\chi}$ stabilizes $T$ and operates identically on $B_{u}, P_{\chi}$ is an 
automorphism of $B$ exactly when its restriction to $T$ is an automorphism of $T . P_{\chi}^{*}: X(T) \rightarrow X(T)$, the endomorphism of the character group dual to $P_{\chi}: T \rightarrow T$, has the formula $P_{\chi}^{*}(\beta)=\beta+a_{\beta} \chi$. When $a_{\chi}=0$, one may check that the inverse to $P_{\chi}^{*}$ has the formula $\left(P_{\chi}^{*}\right)^{-1}(\beta)=\beta-a_{\beta} \chi$. When $a_{\chi} \neq 0$, one may check that $\chi$ is not an element in the image of $P_{\chi}^{*}$.

\subsection{Lemma. Let $\beta \in X(T)$. $P_{\chi}\left(\operatorname{Ker}\left(\beta \circ P_{\chi}\right)\right)=\operatorname{Ker} \beta$, when $a_{\chi} \neq-1$.}

Proof. We may regard this statement as equally about characters on $B$, or characters on $T$, since $P_{\chi}$ acts identically on $B_{u}$ and since characters vanish on $B_{u}$. One may check that if $\varphi$ is a surjective endomorphism of $T$, then $\varphi(\operatorname{Ker}(\beta \circ \varphi))=\operatorname{Ker} \beta$. So it will suffice to show that $P_{\chi}: T \rightarrow T$ is surjective. The kernel of $P_{\chi}$ lies in the center of $\mathrm{Gl}_{n}$, and $P_{\chi}$ at a central element

$$
\left(\begin{array}{lllll}
S & & & & 0 \\
& S & \cdot & \\
0 & & & S
\end{array}\right)
$$

has the value

$$
\left(\begin{array}{cccc}
S^{1+a_{x}} & & & \\
\cdot & & & 0 \\
0 & & \cdot & S^{1+a_{x}}
\end{array}\right) .
$$

Hence, $\operatorname{Ker} P_{\chi}$ is finite when $1+a_{\chi} \neq 0$, and so, $P_{\chi}$ is surjective.

Corollary. Let $\lambda \in X(T)$. If $a_{\chi}=0$, then $P_{\chi}(\operatorname{Ker} \lambda)=$ $\operatorname{Ker}\left(\left(P_{\chi}^{*}\right)^{-1}(\lambda)\right)$.

Proof. The corollary follows from the lemma with $\beta=\left(P_{x}^{*}\right)^{-1}(\lambda)$, using the definition of $P_{\chi}^{*}: P_{\chi}^{*}(\beta)=\beta \circ P_{\chi}$.

3.3. Let $K$ be algebraically closed. Let $\lambda$ be a character on the upper triangular subgroup $B$ of $\mathrm{Gl}_{n}(n \geq 3)$.

THEOREM. Ker $\lambda$ is universally observable if and only if the signature $a_{\lambda}$ of $\lambda$ is zero, and $\pm\left.\lambda\right|_{T \cap \mathrm{Sl}_{n}}$ lies in the dominant chamber.

REMARK. $a_{\lambda}=0$ if and only if $\operatorname{Ker} \lambda$ contains the center of $\mathrm{GL}_{n}$.

Proof. Suppose that $a_{\lambda} \neq 0$. Let $b$ be an integer and let $\chi_{b}$ be the character $-2 b \lambda_{1}+b \lambda_{2}$. Since $a_{\chi_{b}}=0, P_{\chi_{b}}$ is a embedding, and, by the 
corollary in $\$ 3.2, P_{\chi_{b}}(\operatorname{Ker} \lambda)=\operatorname{Ker}\left(\left(P_{\chi_{b}}^{*}\right)^{-1}(\lambda)\right)=\operatorname{Ker}\left(\lambda-a_{\lambda} \chi_{b}\right)$. Since $a_{\lambda}$ is non-zero, the coefficients of $\lambda_{1}$ and $\lambda_{2}$ in $\lambda-a_{\lambda} \chi_{b}$ have opposite signs for large $b$, and so, for large $b, \pm\left.\left(\lambda-a_{\lambda} \chi_{b}\right)\right|_{T \cap S 1_{n}}$ lies not in the dominant chamber. Hence, by [4, Theorem 2.3],

$$
\mathbf{S} 1_{n} / \operatorname{Ker}\left(\left.\left(\lambda-a_{\lambda} \chi_{b}\right)\right|_{B \cap S 1_{n}}\right)
$$

is not quasi-affine. Neither is $\mathrm{Gl}_{n} / \operatorname{Ker}\left(\lambda-a_{\lambda} \chi_{b}\right)$ quasi-affine. If it were,

$$
\mathrm{Gl}_{n} / \operatorname{Ker}\left(\left.\left(\lambda-a_{\lambda} \chi_{b}\right)\right|_{B \cap \mathrm{Sl}_{n}}\right)
$$

would be quasi-affine, since $\operatorname{Ker}\left(\left.\left(\lambda-a_{\lambda} \chi_{b}\right)\right|_{B \cap \mathrm{Sl}_{n}}\right)$ is normal in $\operatorname{Ker}\left(\lambda-a_{\lambda} \chi_{b}\right)$. Then $\mathrm{SL}_{n} /\left(\operatorname{Ker}\left(\left.\left(\lambda-a_{\lambda} \chi_{b}\right)\right|_{B \cap \mathrm{Sl}_{n}}\right)\right)$ would be quasi-affine. Therefore, $\operatorname{Ker} \lambda$ is not universally observable when $a_{\lambda} \neq 0$.

Suppose that $\pm\left.\lambda\right|_{T \cap S l_{n}}$ is not in the dominant chamber. Arguing as above, $\mathrm{GL}_{n} / \operatorname{Ker} \lambda$ is already non-quasi-affine.

Conversely, suppose that $\pm\left.\lambda\right|_{T \cap S l_{n}}$ lies in the dominant chamber and that $a_{\lambda}=0$. Since $a_{\lambda}=0$, the kernel of $\lambda$ contains the center of $\mathrm{Gl}_{n}$, evidently, and so, $\operatorname{Ker} \lambda=\left.B_{u} \cdot \operatorname{Ker} \lambda\right|_{T \cap \mathrm{Sl}_{n}} \cdot \operatorname{Center}\left(\mathrm{Gl}_{n}\right)$. In the next section, we show that the characters of $\operatorname{Ker} \lambda$ which appear in $C(\operatorname{Ker} \lambda)$ form a subgroup. Hence, by Proposition $1.4, \operatorname{Ker} \lambda$ is universally observable. (More accurately, $(\operatorname{Ker} \lambda)^{\circ}$ is universally observable. By [1], it follows that $\operatorname{Ker} \lambda$ is universally observable).

4. Let $P$ be the parabolic subgroup of $\mathrm{Sl}_{n}$ which is associated to a character $\chi$ on $T \cap \mathrm{Sl}_{n}\left(T=\right.$ diagonal subgroup of $\left.\mathrm{Gl}_{n}\right) . P$ is generated by $T \cap \mathrm{Sl}_{n}$ and by the one-parameter unipotent subgroups of $\mathrm{Sl}_{n}$ corresponding to the roots $\alpha$ such that the coroot of $\alpha$ has non-negative value at $\chi$. Let $P_{u}$ be the unipotent radical of $P$ and $B$ the upper triangular subgroup of $\mathrm{Sl}_{n}$.

TheOrem. The subgroup $P_{u} \cdot \operatorname{Ker} \chi \cdot \operatorname{Center}\left(\mathrm{Gl}_{n}\right)$ of $\mathrm{Gl}_{n}$ is universally observable.

COROLlaRy. If $\pm \chi$ lies in the dominant chamber, then $B_{u} \cdot \operatorname{Ker} \chi \cdot$ $\operatorname{Center}\left(\mathrm{Gl}_{n}\right)$ is universally observable.

The corollary follows from the Theorem since $B$ is the parabolic subgroup associated to characters in the dominant chamber.

Proof of the Theorem. Suppose that $H$ is a group such that the monoid $S$ of characters of $H$ on $C(H)$ is a group. Let $G$ be a connected group of the form $H \cdot C$ where $H$ is a subgroup of $G$, and $C$ is a central subgroup, 
and where the multiplication map $H \times C \rightarrow G$ is separable. From the last condition, which is equivalent to requiring that the differential of the multiplication map at the neutral element be surjective, it follows that the associated map hy $(H) \otimes \operatorname{hy}(C) \rightarrow \operatorname{hy}(G)$ is surjective (for instance, by [3, $\S 2$, proposition]).

A character $\chi$ on $G$ appears in the operation of $G$ on the one-dimensional modules in $C(G)$ exactly when $\left.\chi\right|_{H}$ appears in the operation of $H$ on $C(G)$, since $C$ operates trivially on hy $(G)$. Furthermore, the operations of $H$ on $C(H)$ and on $C(G)$ give the same monoid of characters on $H$. In fact, any $H$-submodule of hy $(G)$ is a quotient of a direct sum of $H$-submodules of hy $(H)$, since hy $(H) \otimes \operatorname{hy}(C) \rightarrow \operatorname{hy}(G)$ is surjective, and since $C(H)$ is closed under quotients by Lemma 1.2.

Taking $H=P_{u} \cdot \operatorname{Ker} \chi$ and $C=\operatorname{Center}\left(\mathrm{Gl}_{n}\right)$ (which satisfies the hypothesis of separability since $\mathrm{Sl}_{n} \cdot C$ does), we will show that $P_{u} \cdot \operatorname{Ker} \chi$ - Center $\left(\mathrm{Gl}_{n}\right)$ is universally observable by showing that the monoid of characters of $P_{u} \cdot \operatorname{Ker} \chi$ on $C\left(P_{u} \operatorname{Ker} \chi\right)$ is a group, and hence, so is the monoid of characters of $\boldsymbol{P}_{u} \cdot \operatorname{Ker} \chi \cdot \operatorname{Center}\left(\mathrm{Gl}_{n}\right)$ on

$$
C\left(P_{u} \cdot \operatorname{Ker} \chi \cdot \operatorname{Center}\left(\mathrm{Gl}_{n}\right)\right)
$$

by the previous paragraph.

Let $W$ be the Weyl group of $\mathrm{Sl}_{n}$ relative to $T \cap \mathrm{Sl}_{n}$, an $w$ an element which conjugates $B$ into $P$. If we replace $\chi$ by its transform under $w^{-1}, \chi$ will be a dominant weight relative to $B . \chi$ has the form $\sum_{i \in J} n_{i} \lambda_{i}, n_{i}>0$, for some subset $J$ of $\{1, \ldots, n\} . P_{u}$ is generated by one parameter subgroups $G_{\alpha}$, the coroot of $\alpha$ being positive at $\chi$, and $L\left(P_{u}\right)$ is spanned by $\left\{E_{j k} \mid j \leq i<k\right.$ for some $\left.i \in J\right\}$, where $E_{j k}$ is the matrix whose single non-zero entry is a 1 in the $j k$ th position.

Let $A$ be the group of characters generated by

$$
\left\{\left.\left(\alpha_{j}+\cdots+\alpha_{k-1}\right)\right|_{P_{u} \operatorname{Ker} \chi} \mid j \leq i<k \text { for some } i \in J\right\} .
$$

The character monoid $M$ for $P_{u} \operatorname{Ker}_{\chi}$ relative to $C\left(P_{u} \mathrm{Ker}_{\chi}\right)$ is a submonoid of $A$, since $\alpha_{j}+\cdots+\alpha_{k-1}$ is the weight of $T \cap \mathrm{Sl}_{n}$ operating on $K E_{j k} \subset L\left(P_{u} \operatorname{Ker} \chi\right)$. We will know $M$ for a group once we show that $M$ contains a subgroup of finite index in $A$.

Fix $i$ in $J$, and take the highest non-zero exterior power of the $P_{u}$ Ker $\chi$-module spanned by the $E_{j k}, j \leq i<k$. Since $\alpha_{j}+\cdots+\alpha_{k-1}$ is the weight of $E_{j k}, a_{l}$ is a term in the weight of $E_{j k}$ exactly when $j \leq l<k$. Hence, the $T \cap \mathrm{Sl}_{n}$-weight of the exterior power is $(n-i) \sum_{j=1}^{i} j \alpha_{j}+$ $i \sum_{j=i+1}^{n-1}(n-j) \alpha_{j}$, which equals $n \lambda_{i}$.

Since $\chi$ is zero on $P_{u} \operatorname{Ker} \chi,-n n_{i} \lambda_{i}$ equals $\sum_{j \neq i} n n_{j} \lambda_{j}$, which is an element of $M$. That is, $-n_{i}\left(\sum_{j \leq i<k} \operatorname{wt}\left(E_{j k}\right)\right)$ is an element of $M$, for any $i$ 
in $J$. Evidently, $w t\left(E_{j^{\prime} k^{\prime}}\right), j^{\prime} \leq i<k^{\prime}$, lies in $M$. Hence

$$
-n_{i}\left(\mathrm{wt}\left(E_{j k}\right)\right)=-n_{i}\left(\sum_{j^{\prime} \leq i<k^{\prime}} \operatorname{wt}\left(E_{j^{\prime} k^{\prime}}\right)\right)+n_{i}\left(\sum_{\substack{j^{\prime} \leq i<k^{\prime} \\\left(j^{\prime}, k^{\prime}\right) \neq(j, k)}} \operatorname{wt}\left(E_{j^{\prime} k^{\prime}}\right)\right)
$$

lies in $M$. Therefore, $M$ contains the subgroup (of finite index) of $A$ generated by

$$
\left\{\left.\left(\prod_{i \in J} n_{i}\right)\left(\alpha_{j}+\cdots+\alpha_{k-1}\right)\right|_{P_{u} \operatorname{Ker} \chi} \mid j \leq i<k \text { for some } i \in J\right\} .
$$

\section{REFERENCES}

[1] Bialynicki-Birula, Hochschild, and Mostow, Extensions of representations of algebraic linear groups, Amer. J. Math., 85 (1963), 131-144.

[2] G. Hochschild, Introduction to Affine Algebraic Groups, Holden-Day, San Francisco, 1971.

[3] J. Sullivan, Repesentations of the hyperalgebra of an algebraic group, Amer. J. Math., 100 (1978), 643-52.

[4] J. Sullivan, Quasi-affine homogeneous spaces, J. Algebra, 77, No. 2, (1982), 544-551.

[5] M. Sweedler, Unpublished notes on universal observability.

Received April 4, 1984. Supported in part by National Science Foundation Grant MCS 77-18723(02) at the Institute for Advanced Study.

UNIVERSITY OF WASHINGTON

SEATTLE, WA 98195 



\section{PACIFIC JOURNAL OF MATHEMATICS EDITORS}

V. S. VARAdarajan (Managing Editor)
University of California
Los Angeles, CA 90024
CHARLES R. DePrima
California Institute of Technology
Pasadena, CA 91125
R. FINN
Stanford University
Stanford, CA 94305

V. S. VARADARAJAN (Managing Editor)

Hermann FlaschKa

University of Arizona

Tucson, AZ 85721

RAMESH A. GANGOlli

University of Washington

Seattle, WA 98195

ROBION KIRBY

University of California

Berkeley, CA 94720
C. C. MOORE

University of California

Berkeley, CA 94720

H. SAMELSON

Stanford University

Stanford, CA 94305

Harold Stark

University of California, San Diego

La Jolla, CA 92093

\section{ASSOCIATE EDITORS}
R. ARENS
E. F. BECKENBACH
B. H. NeumanN
F. WOLF
K. Yoshida (1906-1982)

\section{SUPPORTING INSTITUTIONS}

\author{
UNIVERSITY OF ARIZONA \\ UNIVERSITY OF BRITISH COLUMBIA \\ CALIFORNIA INSTITUTE OF TECHNOLOGY \\ UNIVERSITY OF CALIFORNIA \\ MONTANA STATE UNIVERSITY \\ UNIVERSITY OF NEVADA, RENO \\ NEW MEXICO STATE UNIVERSITY \\ OREGON STATE UNIVERSITY
}

UNIVERSITY OF OREGON UNIVERSITY OF SOUTHERN CALIFORNIA

STANFORD UNIVERSITY

UNIVERSITY OF HAWAII

UNIVERSITY OF TOKYO

UNIVERSITY OF UTAH

WASHINGTON STATE UNIVERSITY

UNIVERSITY OF WASHINGTON 


\section{Pacific Journal of Mathematics}

\section{Vol. 120, No. $1 \quad$ September, 1985}

Ulrich F. Albrecht, A note on locally $A$-projective groups $\ldots \ldots \ldots \ldots \ldots 1$

Marilyn Breen, A Krasnosel'skiü-type theorem for unions of two starshaped

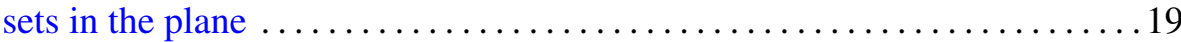

Anthony Carbery, Sun-Yung Alice Chang and John Brady Garnett,

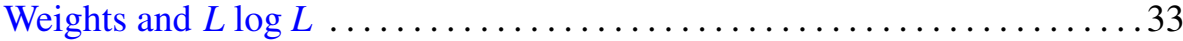

Joanne Marie Dombrowski, Tridiagonal matrix representations of cyclic

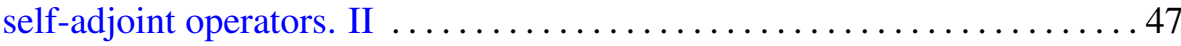

Heinz W. Engl and Werner Römisch, Approximate solutions of nonlinear random operator equations: convergence in distribution $\ldots \ldots \ldots \ldots \ldots 55$

P. Ghez, R. Lima and J. E. Roberts, $W^{*}$-categories $\ldots \ldots \ldots \ldots \ldots \ldots$

Barry E. Johnson, Continuity of homomorphisms of Banach $G$-modules . . 111 Elyahu Katz and Sidney Allen Morris, Free products of topological groups

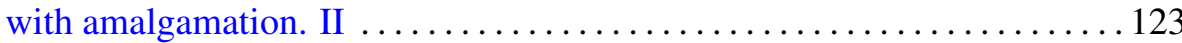

Neal I. Koblitz, $p$-adic integral transforms on compact subgroups of $\mathbf{C}_{p} \ldots 131$ Albert Edward Livingston, A coefficient inequality for functions of positive real part with an application to multivalent functions $\ldots \ldots \ldots 139$

Scott Carroll Metcalf, Finding a boundary for a Hilbert cube manifold

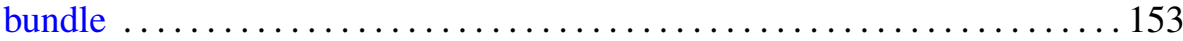

Jack Ray Porter and R. Grant Woods, When all semiregular $H$-closed extensions are compact ........................

Francisco José Ruiz and José Luis Torrea, A unified approach to Carleson measures and $A_{p}$ weights. II $\ldots \ldots \ldots \ldots \ldots \ldots \ldots \ldots \ldots \ldots \ldots \ldots \ldots$

Timothy DuWayne Sauer, The number of equations defining points in general position

John Brendan Sullivan, Universal observability and codimension one subgroups of Borel subgroups

Akihito Uchiyama, Extension of the Hardy-Littlewood-Fefferman-Stein inequality 